\title{
Intestinal Microbiota: An Emerging Target for Modifying Cardiovascular Health
}

Shalin S Patel ${ }^{1}$, Mazyar Malakouti' ${ }^{2}$, Salil Sethi ${ }^{1}$ and Robert J Chilton ${ }^{1 *}$

${ }^{1}$ Division of Cardiology, The University of Texas Health Science Center at San Antonio, San Antonio, Texas, USA

${ }^{2}$ Department of Medicine, The University of Texas Health Science Center at San Antonio, San Antonio, Texas, USA

\begin{abstract}
The rising prevalence of obesity and Type 2 diabetes mellitus (T2DM) worldwide is a primary public health concern as it heavily impacts risk of cardiovascular disease (CVD) and other comorbid diseases. Promising research in the last decade implicates gut microbiota as an important contributor to risk of developing obesity, T2DM and CVD. Animal models of obesity and insulin resistance identify that germ free mice have decreased risk of obesity and insulin resistance despite being on high fat sugar rich diet compared to conventional mice. Obesity is shown to be transmissible through faecal microbiome transplantation from obese phenotype mice to germ free mice. There is also growing evidence that gut microbiota metabolism of choline to Trimethylamine-N-Oxide (TMAO) is directly linked to increased cardiovascular risk. TMAO is a proatherogenic molecule which has been shown to increase atherosclerotic plaque burden in murine atherosclerosis model. Also, clinical cohort studies have established increased TMAO levels as an independent predictor for major adverse cardiovascular events including death, myocardial infarction and stroke. As it stands, intestinal microbiota-mediated pathogenesis of obesity, T2DM and CVD is still an emerging research direction and has the potential for leading to new diagnostic and therapeutic strategies.
\end{abstract}

Keywords: Cardiovascular disease; Atherosclerosis; Gut microbiome; Insulin resistance; Obesity; Choline; Trimethylamine $\mathrm{N}$-oxide

\section{Introduction}

Cardiovascular disease (CVD) remains the largest cause of morbidity and mortality in the developed world. Management of risk factors for CVD continues to be the primary prevention strategy to lower the incidence of CVD. One of the foremost challenges to CVD prevention is the rising prevalence of obesity and Type 2 diabetes (T2DM) worldwide, which are potent risk factors for development of atherosclerosis. The multifaceted relationship of atherosclerosis, obesity and Type 2 diabetes involves numerous genetic, nutritional, and environmental determinants [1]. Dietary modification is currently the primary preventative approach to reduce incidence of cardiometabolic syndrome with emphasis on low fat, carbohydrate and cholesterol content coupled with caloric restriction. Recent research has shown that dietary phospholipid and carbohydrate metabolism by intestinal microbiota or 'gut flora' is involved in cardiometabolic syndrome and CVD [2].

Human intestinal microbiota comprises of over 100 trillion distinct organisms with more than 1,000 distinct species and approximately 5 million microbial genes [3,4]. Gut colonization begins at birth and stabilizes at age 3-4 yrs. There is considerable variability in microbiota composition between individuals, however most communities are dominated with species belonging to the Bacteroidetes, Firmicutes, Actinobacteria, Proteobacteria and Verrucomicrobio phyla [5]. Microbiota composition can also be altered with host age, diet and metabolism [5,6]. Specifically, proportion of Bifidobacteria species in fecal samples reduces significantly in healthy elderly subjects compared to healthy children and young adults. However, there is limited variation in anaerobic microbiota composition between healthy cohorts [6]. In contrast, there is considerable variation in gut microbiota composition in subjects with obesity and T2DM [7]. There is increasing recognition of the complex role of intestinal microbiota in modifying systemic inflammation, energy harvest and promotion of atherogenesis [4]. Understanding the mechanistic link of host-microbiota relationship has the promise for developing new preventative and therapeutic strategies.

\section{Intestinal Microbiota: Effect on Obesity and T2DM}

Murine animal models indicate a link between gut flora composition and risk of developing obesity and insulin resistance [2]. Caecal microbiome transplantation from obesity phenotype mice into germ free mice resulted in successful transmission of obesity trait with increased total body fat content [8]. Additionally, germ free mice fed with high fat-sugar rich diet were noted to have less total body fat content compared to conventionally caged mice with similar dietary caloric intake. However, when the same germ free mice were exposed to conventional caecal microbiota, there was a significant increase in total body fat content [9]. Microbiota composition is also linked to changes in weight and obesity. Genetically hyperphagic obese ${ }^{\mathrm{ob} / \mathrm{ob}}$ mice had increased proportion of Firmicutes species and diminished counts of Bacteroidetes in caecal microbiota compared to lean wild type controls [7]. Levels of Bacteroidetes also increase in individuals with caloric restriction or weight loss after Roux-en-Y gastric bypass operation [10] More robust nutrient extraction through readily absorbed Short Chain Fatty Acids (SCFAs) is proposed as the underlying mechanism for gut microbiome mediated obesity [8]. There is increased generation of Short-Chain Fatty Acids (SCFAs) e.g. acetate, butyrate, etc by intestinal microbiota through breakdown of non-digestible carbohydrates. SCFAs serve as an energy source for the host and also modify numerous signaling pathways involved in appetite and energy metabolism [9]. The

*Corresponding author: Dr. Robert J. Chilton, Division of Cardiology, Department of Medicine, University of Texas Health Science Center at San Antonio, 7703 Floyd Curl Drive, San Antonio, TX 78229, USA, Tel: 210-279-1248; E-mail: chiltonr@gmail.com

Received September 16, 2014; Accepted October 29, 2014; Published October 31,2014

Citation: Patel SS, Malakouti M, Sethi S, Chilton RJ (2014) Intestinal Microbiota: An Emerging Target for Modifying Cardiovascular Health. J Diabetes Metab 5: 459 doi:10.4172/2155-6156.1000459

Copyright: ( 2014 Patel SS, et al. This is an open-access article distributed unde the terms of the Creative Commons Attribution License, which permits unrestricted use, distribution, and reproduction in any medium, provided the original author and source are credited. 
complex interplay between SCFAs and gut microbiome is determined by the composition of specific bacterial taxa and daily dietary fiber intake by the host [11]. Caecal microbiota enriched with Lactobacillus gasseri and Streptococcus mutans are associated with impaired glucose tolerance and T2DM subjects [12]. Conversely, metagenomic studies on faecal microbiome of normal, impaired glucose and T2DM subjects indicated lower levels of Faecalibacterium prausnitzii to be associated with T2DM, which is a butyrate SCFA producing species [13]. Butyrate SCFA producing species have been to reduce plasma glucose levels by enhancing GLP-1 release, leptin synthesis and overall reduction in insulin resistance [12]. SCFAs also alter intestinal permeability leading to a chronic inflammatory state [2]. There is a direct relationship with translocation of intestinal bacteria and activation of inflammatory cascade through macrophage activation by bacterial endotoxins e.g. lipopolysaccharide (LPS) [4]. Chronic low dose LPS exposure in mice has been shown to promote IR, obesity, dyslipidemia and hepatic steatosis through toll-like receptors (TLRs) [4]. This is further validated in mouse model with TLR4 deficient mice, who display resistance to obesity despite being on a high fat diet [14]. However, there is conflicting evidence on microbiota composition and its relationship to obesity and DM. Numerous environmental variables including diet impact microbiota composition and limitations in complete characterization of microbiome continues to represent a challenge in establishing a clear link between unique microbiomes and disease. At present, there is insufficient evidence to make clear causal relationship between specific intestinal microbiome to risk of obesity and T2DM [15].

\section{Intestinal microbiota and cardiovascular disease}

The influence of gut flora on host lipid metabolism and atherogenesis has been recently characterized through landmark rigorous metabolomic studies of plasma from patients with known, but stable atherosclerotic disease and compared them to analytes from matched controls [16]. Wang et al. identified three distinct phospholipid metabolites of interest - choline, betaine and Trimethylamine-N-Oxide (TMAO) through metabolomic analysis and demonstrated that these metabolites were independent predictors for clinical cardiovascular risk. Choline is a semi-essential nutrient which is present in egg, liver, poultry and fish as dietary phosphatidylcholine (PC), the predominant phospholipid found in cell membranes [17]. It is also a precursor to the neurotransmitter acetylcholine and deficiencies are associated with neurological impairment. Animals and humans obtain choline from dietary conversion of phospatidylcholine by Phospolipase D. Betaine is obtained from ingestion or through choline oxidation in the kidney and liver. Intestinal microbiota metabolize choline and betaine in the gut lumen to produce gaseous Trimethylamine (TMA) $[1,16]$. Many oral and gut bacterial commensals are implicated in conversion of choline to TMA in the gut, including Streptococcus, Clostridia, Proteus, Shigella and Aerobacter [1]. TMA is readily reabsorbed across the intestinal epithelium and into host plasma. Subsequently, it is rapidly oxidized to Trimethylamine-N-oxide (TMAO) by group of Flavin Monooxygenases (FMO) in the liver [16,18]. FMO3 is the predominant enzyme responsible for nearly $90 \%$ conversion of TMA to TMAO [16]. Dietary L-carnitine found largely in red meat is another precursor for gut flora mediated TMA and TMAO synthesis [13]. Koeth et al. demonstrated that L-carnitine challenge test in omnivorous human subjects being screened for CVD had increased levels of TMA and TMAO compared to vegan and vegetarian controls. Comparison of human faecal microbial composition found that Clostridiaceae and Peptostreptococcaceae species positively correlated with an omnivorous diet and TMAO production [19]. Interestingly, similar L-carnitine challenge in vegan subjects did not increase TMAO levels [19]. These findings further support that dietary habits are an essential determinant of gut microbiota composition and TMAO synthesis. By extension, the results also explain the increased risk of CVD associated with high red meat intake.

TMAO is a proatherogenic molecule which is directly involved in atherosclerosis. In murine atherosclerotic model, apoE $\%$ mice were fed choline and TMAO supplemented diets. Subsequently, TMAO levels were measured which directly related to increasing atherosclerotic plaque burden. TMAO supplementation in mice also enhances macrophage uptake of cholesterol and promotes the proliferation of foam cells in atherosclerosis through stimulation of scavenger receptors CD36 and SRA1 [16]. These studies were extended recently to a large clinical cohort study at Cleveland Clinic to assess association of TMAO with major adverse cardiovascular events including myocardial infarction, stroke and cardiovascular death. Plasma levels of TMAO were measured for 4007 patients undergoing elective diagnostic cardiac catheterization at Cleveland clinic. Subsequently they were followed clinically for 3 years. Results from trial indicated an independent association between elevated TMAO levels and increased risk of incident major adverse cardiovascular events. Even in low risk subgroups, the prognostic value of TMAO remained significant for increased MACE rate [20]. These observations were extended with follow up study on the same clinical cohort to assess whether elevated choline and betaine plasma levels were associated with poor cardiovascular prognosis. Interestingly, choline and betaine levels are positively associated with poor cardiovascular prognosis only in the presence of coexisting elevated TMAO levels. Therefore, TMAO synthesis by gut microbiota is likely the major prognostic factor in development of cardiovascular events rather than its precursors [17].

\section{Intestinal microbiota: Therapeutic Modification}

Aforementioned animal and human studies highlight the intricate contribution of gut microbiota in modifying risk for obesity, insulin resistance and atherosclerosis (Figure 1). Targeting the effects of gut microbiota or microbiome composition represents a novel approach to reducing incident cardiovascular event rates. Antibiotic mediated suppression of intestinal microbiota and by extension TMAO levels has been studied in mouse models. Mice on high choline diet were exposed to broad spectrum antibiotics compared to control showed lower TMAO levels and lower average atherosclerotic plaque areas [16] Small studies in humans have shown lower TMAO levels associated with antibiotic suppression of gut flora [20]. However, there are no large prospective studies to assess the effects of gut flora suppression on development of metabolic syndrome or cardiovascular events. Additionally, long term administration of antibiotic therapy carries risk of proliferation of antibiotic resistant gut flora/pathogens [15]. Probiotics are an attractive alternative to chronic antimicrobial therapy in modifying intestinal microbiota composition. Decreased levels of choline and trimethylamines are noted in mice supplemented with probiotic formulation of Lactobacillus paracasei; however, there was no significant change in liver TMAO or plasma choline levels [21]. Metaanalysis of small trials of probiotic administration of Lactobacillus acidophilus in humans reveals mixed results on modifying lipid profiles [22]. Currently, there are no studies that indicate prevention or reversal of atherosclerosis with probiotic administration $[4,23]$.

\section{Intestinal microbiota: Looking forward}

As it stands, this is an emerging research segment which continues to require additional investigation to truly assess the role of gut microbiota composition in cardiometabolic syndrome and 


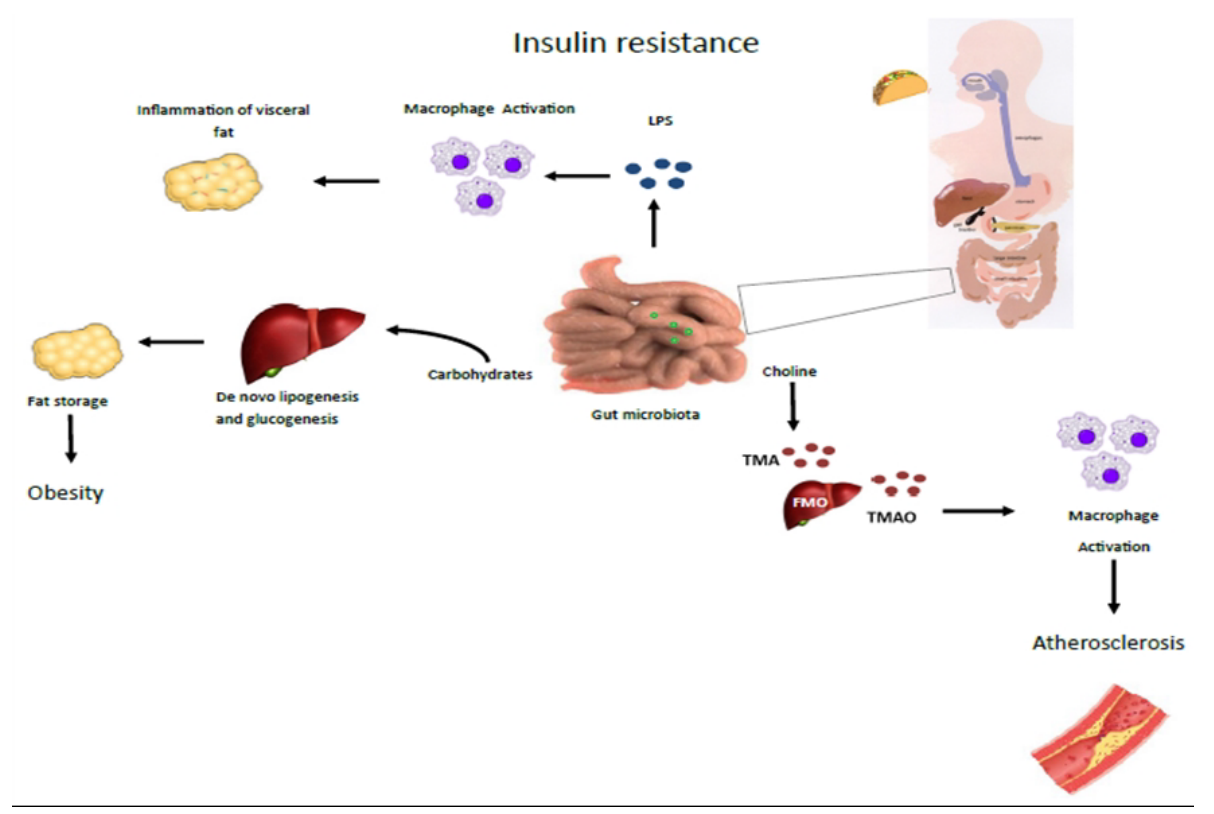

Figure 1: Major gut flora mediated mechanisms leading to cardiometabolic syndrome. 1. Atherosclerosis pathway depicted by choline conversion to TMA in the gut by microbiota and subsequent conversion to TMAO in liver. Increased TMAO levels lead to macrophage activation and plaque formation. 2. Obesity pathway depicts conversion of carbohydrates to SCFA which enhances gluconeogenesis and lipid accumulation. 3. Insulin resistance pathway is depicted with SCFA mediated endotoxemia with LPS by increased membrane permeability. Macrophage activation is promoted by LPS endotoxemia along with chronic inflammatory state and leads to increased insulin resistance. (Modified: Vinje S, Stroes E, Nieuwdorp M, Hazen SL (2014) The gut microbiome as novel cardiometabolic target: the time has come! Eur Heart J 35: 883-887).

CVD. Identification of specific bacterial taxa or gut composition that promote cardiometabolic syndrome and atherogenesis will be crucial in development of therapeutic interventions by use of targeted antibiotics, microbiome transplantation or probiotics [23]. Further exploration of the metabolic pathway leading to TMAO offers the most promising mechanism for therapies. Genomic targeting of FMO3 in animal model of atherosclerosis and by extension, TMAO synthesis can provide greater insights [24]. However, current data suggests the importance of advocating avoidance of choline rich diet and correspondingly increasing vegetable and fruit intake relative to red meat and eggs.

\section{Conclusion}

Recent research implicates gut microbiota composition as an important risk contributor for developing obesity, T2DM and CVD. Observational and interventional studies in animal and human models confirm a potent correlation between diets rich in choline, trimethylamines with cardiovascular disease. Discovery of gut microbiota generated proatherogenic TMAO molecule from choline and L-carnitine further highlights the obligate role of intestinal microbiome in human pathogenesis. Establishment of an independent link between elevated TMAO levels and CVD risk has provided insights into metaorganismal origin of disease. As such, advancements in targeting metabolic pathway for TMAO synthesis and elucidating specific microbial taxa involved in cardiometabolic syndrome has potential for novel therapies. These findings also support the intricate relationship between multiple factors in the pathogenesis of cardiometabolic syndrome.

\section{References}

1. Loscalzo J (2011) Lipid metabolism by gut microbes and atherosclerosis. Circ Res 109: 127-129.

2. Kootte RS, Vrieze A, Holleman F, Dallinga-Thie GM, Zoetendal EG, et al.
(2012) The therapeutic potential of manipulating gut microbiota in obesity and type 2 diabetes mellitus. Diabetes Obes Metab 14: 112-120.

3. Qin J, Li R, Raes J, Arumugam M, Burgdorf KS, et al. (2010) A human gut microbial gene catalogue established by metagenomic sequencing. Nature 464: 59-65.

4. Vinjé S, Stroes E, Nieuwdorp M, Hazen SL (2014) The gut microbiome as novel cardio-metabolic target: the time has come! Eur Heart J 35: 883-887.

5. Knaapen M, Kootte RS, Zoetendal EG, de Vos WM, Dallinga-Thie GM, et al. (2013) Obesity, non-alcoholic fatty liver disease, and atherothrombosis: a role for the intestinal microbiota? Clin Microbiol Infect 19: 331-337.

6. Hopkins MJ, Sharp R, Macfarlane GT (2002) Variation in human intestinal microbiota with age. Dig Liver Dis 34 Suppl 2: S12-18.

7. Ley RE, Bäckhed F, Turnbaugh P, Lozupone CA, Knight RD, et al. (2005) Obesity alters gut microbial ecology. Proc Natl Acad Sci U S A 102: 11070 11075.

8. Turnbaugh PJ, Ley RE, Mahowald MA, Magrini V, Mardis ER, et al. (2006) An obesity-associated gut microbiome with increased capacity for energy harvest. Nature 444: 1027-1031.

9. Bäckhed F, Manchester JK, Semenkovich CF, Gordon JI (2007) Mechanisms underlying the resistance to diet-induced obesity in germ-free mice. Proc Nat Acad Sci U S A 104: 979-984.

10. Tremaroli V, Bäckhed $F$ (2012) Functional interactions between the gut microbiota and host metabolism. Nature 489: 242-249.

11. den Besten G, van Eunen K, Groen AK, Venema K, Reijngoud DJ, et al. (2013) The role of short-chain fatty acids in the interplay between diet, gut microbiota, and host energy metabolism. J Lipid Res 54: 2325-2340.

12. Karlsson FH, Tremaroli V, Nookaew I, Bergström G, Behre CJ, et al. (2013) Gut metagenome in European women with normal, impaired and diabetic glucose control. Nature 498: 99-103.

13. Cani PD, Amar J, Iglesias MA, Poggi M, Knauf C, et al. (2007) Metabolic endotoxemia initiates obesity and insulin resistance. Diabetes 56: 1761-1772.

14. Sanz Y, Santacruz A, Gauffin P (2010) Gut microbiota in obesity and metabolic disorders. Proc Nutr Soc 69: 434-441. 
Citation: Patel SS, Malakouti M, Sethi S, Chilton RJ (2014) Intestinal Microbiota: An Emerging Target for Modifying Cardiovascular Health. J Diabetes Metab 5: 459 doi:10.4172/2155-6156.1000459

15. Tilg $H$, Kaser A (2011) Gut microbiome, obesity, and metabolic dysfunction. J Clin Invest 121: 2126-2132.

16. Wang Z, Klipfell E, Bennett BJ, Koeth R, Levison BS, et al. (2011) Gut flora metabolism of phosphatidylcholine promotes cardiovascular disease. Nature 472: 57-63.

17. Wang Z, Tang WH, Buff JA, Fu X, Britt EB, et al. (2014) Prognostic value of choline and betaine depends on intestinal microbiota-generated metabolite trimethylamine-N-oxide. Eur Heart J 35: 904-910.

18. Bennett BJ, De Aguiar Vallim TQ, Wang Z, Shigh DM, Meng Y, Gregory J, et al (2013) Trimethylamine-N-oxide, a metabolite associated with atherosclerosis, exhibits complex genetic and dietary regulation. Cell Metabolism 17:49-60.

19. Koeth RA, Wang Z, Levison BS, Buffa JA, Org E, et al. (2013) Intestinal microbiota metabolism of L-carnitine, a nutrient in red meat, promotes atherosclerosis. Nat Med 19: 576-585.
20. Tang WH, Wang Z, Levison BS, Koeth RA, Britt EB, et al. (2013) Intestinal microbial metabolism of phosphatidylcholine and cardiovascular risk. N Engl J Med 368: 1575-1584

21. Martin FP, WangY, Sprenger N, Yap IK, Lundstedt T, et al. (2008) Probiotic modulation of symbiotic gut microbial-host metabolic interactions in a humanized microbiome mouse model. Mol Syst Biol 4: 157.

22. DiRienzo DB (2014) Effect of probiotics on biomarkers of cardiovascular disease: implications for heart-healthy diets. Nutr Rev 72: 18-29.

23. Brown JM, Hazen SL (2014) Metaorganismal nutrient metabolism as a basis of cardiovascular disease. Curr Opin Lipidol 25: 48-53.

24. Hartiala J, Bennett BJ, Tang WH, Wang Z, Stewart AF, et al. (2014) Comparative genome-wide association studies in mice and humans for trimethylamine $\mathrm{N}$-oxide, a proatherogenic metabolite of choline and L-carnitine. Arterioscle Thromb Vasc Biol 34: 1307-1313. 